\title{
El uso de la traducción para mejorar la comprensión lectora de textos especializados en los estudiantes universitarios
}

The use of translation to improve reading comprehension of specialized texts in university students

Rocío de los Ángeles Barragán Murillo ${ }^{1}$

\section{Resumen}

El objetivo de este trabajo es proponer la utilización de actividades de traducción en las aulas de clase para mejorar la comprensión lectora de textos especializados en inglés para estudiantes universitarios. Se ha realizado una revisión bibliográfica que sustenten la utilización de la traducción, a su vez, se ha determinado a través de conceptos y definiciones las características de los textos especializados que los estudiantes deben comprender como parte de su actividad formativa. Por lo tanto, se determina que su uso es aconsejable.

Palabras clave: Traducción, lectura, comprensión, textos especializados.

\section{Abstract}

This work aims at proposing the use of translation activities in the English classroom to improve the reading comprehension. The literature review was carried out to support the use

\footnotetext{
${ }^{1}$ Escuela Superior Politécnica de Chimborazo, robarragan@espoch.edu.ec
} 
of translation. The concepts, definitions, and features of specialized texts that students must learn to understand as part of their academic activities were determined. Therefore, the use of translation is advisable.

Keywords: Translation, Reading, comprehension, specialized texts.

\section{Introducción}

El éxito de los estudiantes universitarios depende del dominio de destrezas como leer, escribir, escuchar, hacer presentaciones y razonar. Por lo tanto, la educación universitaria requiere que los estudiantes desarrollen diferentes estrategias de lectura. La habilidad de leer textos académicos se considera como una de las destrezas más importantes en los estudiantes universitarios cuya lengua materna no es inglés. Haremos referencia a la traducción no de tipo profesional sino más bien una de tipo pedagógica para mejorar las destrezas lectoras en textos especializados. La sola idea de que los estudiantes traduzcan para comprender una lectura, escribir un texto o hablar causa rechazo en la mayoría de los maestros de inglés quienes continúan en búsqueda de métodos que facilitan el proceso de enseñanza aprendizaje del idioma inglés. Los estudiantes universitarios en muchas ocasiones no pueden comprender un texto que está escrito en inglés general. La traducción, en este caso, es una actividad que ayuda a facilitar la comunicación interpretando la información recibida de un idioma a otro y viceversa. La función básica de la traducción es transmitir de manera apropiada el significado de una palabra u oración de manera lingüística, semántica y pragmática.

\section{La traducción}

La traducción desempeña un rol importante en la sociedad moderna porque permite que las ideas, los conocimientos y la información fluyan entre las diferentes culturas. Sin ella, la ciencia y la tecnología no podrían avanzar. Vivimos en una era que se ha denominado como sociedad de la información, en tal virtud, la traducción científica y técnica es mucho más importante. La traducción ha facilitado una gran cantidad de avances tecnológicos y científicos que han cambiado nuestra forma de vivir. Sin duda, ha acompañado estas invenciones como instrumento de difusión del conocimiento científico y técnico. (Byrne, 2012) 
La traducción es tan antigua como la escritura. Cuando hablamos de la historia de la traducción, inmediatamente pensamos en los textos bíblicos, pero no son los únicos, también se puede hablar de la traducción científica, la cual ha acompañado cada descubrimiento científico y tecnológico a través del tiempo. No existe ningún invento que no haya sido traducido a otra lengua y cultura. Siempre ha facilitado la difusión del conocimiento, pero no fue hasta el siglo XV que alcanzo su plenitud con el invento de la prensa, que revoluciono la escritura y permitió la impresión de libros. Este invento permitió difundir el conocimiento científico y técnico en toda Europa. Durante el renacimiento se escribieron un sinnúmero de libros en medicina, agricultura, zootecnia, pesca, halconería, ciencias militares, navegación, ingeniería, carpintería, fabricación de vidrio, etc. Con la prensa, se facilitó la reproducción de textos en la lengua original y también la traducción de la información. De este modo, la traducción contribuyo con la difusión del conocimiento por siglos. No fue sino hasta hace unos 100 años atrás que la traducción cobro auge. Los investigadores no solo realizaban innumerables inventos, sino que también escribían sobre ellos en su lengua materna, por tanto, la demanda por las traducciones de estas investigaciones era demasiado alta. Gracias a la traducción, los investigadores pudieron acceder a los descubrimientos de sus colegas, es así que la comunidad científica pudo compartir la información y como resultado se produjeron más descubrimientos.

Según Byrne (2012) la traducción es una fuerza importante accionante de la sociedad moderna, la cual facilita el flujo de las ideas, experticia, valores e información entre diferentes culturas. Además, señala que es esencial en los avances tecnológicos y científicos. Mientras que para Leonardi (2010) la traducción es una comunicación, es decir, una forma de comunicación multicultural. Así mismo, indica que existen numerosas definiciones y teorías, y que, para muchos autores, la traducción es una actividad lingüística mecánica que tiene como objetivo remplazar elementos léxicos y morfo sintácticos de una lengua a otra. Afirma que la traducción permite que las personas estén en contacto con el resto del mundo, y que, si no existiese, las comunidades del mundo estarían condenadas a un aislamiento cultural. Por otro lado, Ghazala (1995) la traducción se usa de manera general para referirse a los procesos y los métodos que se utilizan para convertir el significado de la lengua origen a la lengua meta. Para este autor, el significado es un elemento esencial en la traducción. El significado del texto origen es esencial para encontrar el equivalente apropiado en el texto 
meta. El significado en relación a gramática, estilo y sonido. Catford (1995) indica que la traducción es el intento de reemplazar el material textual de la lengua origen por un equivalente textual en la lengua meta.

Es necesario diferenciar la traducción profesional de la traducción pedagógica. La primera tiene como objetivo enseñar a los estudiantes de traducción profesional a traducir, mientras que la segunda tiene como objetivo utilizar la traducción como medio para mejorar las destrezas lingüísticas (Lonsdale, 1993). Para entender la traducción con fines educacionales, empezaremos con la amplia definición que hace Richard W. Brislin sobre traducción. Para este autor, la traducción es el término que se refiere a la transferencia de pensamientos e ideas de la lengua origen a la lengua meta. La traducción pedagógica se encuentra en sus fases iniciales, dado que los maestros de inglés continúan buscando métodos que les permita proporcionar una educación sistemática a aquellos que no son traductores profesionales. La actividad de traducción tiene un rol muy importante como recurso didactico en las universidades europeas, pero no debe girar sobre ella el proceso de esneñanza-aprendizaje del idioma ingles (Hernández, 1996).

Diremos entonces que, la traducción pedagógica es el uso de la traducción con el fin de enseñar un idioma. La traducción pedagógica a diferencia de la traducción literaria no busca perfección artística. Para Shei y Gao (2017) la traducción pedagógica se refiere a la utilización de la traducción con fines pedagógicos, es decir, mejorar las destrezas de escribir, hablar, escuchar y leer que se requieren en la adquisición de una segunda lengua. Este concepto difiere de la enseñanza de la traducción para traductores profesionales. De acuerdo a Loranc-Pasylk (2019) es necesario enfatizar en que la utilización de la traducción es un recurso didáctico y no es el objetivo final de las actividades que se realizan en clase. La función del texto traducido es ser un instrumento para mejorar la competencia del idioma, la concienciación, la práctica, la evaluación del conocimiento del idioma y la memorización.

Mahmoud (2006) citando a (Bonyadi 2003; Owen 2003) describe principios que apoyan el uso de la traducción en clase: La traducción usa material autentico, es interactiva, está centrada en el estudiante y fomenta la autonomía del estudiante. La traducción en el proceso de enseñanza aprendizaje del idioma ingles se considera aceptable. Este tipo de traducción centrada en el estudiante se diferencia del método grammar-translation que es un método 
muy antiguo. Se caracteriza porque las clases se desarrollan en su mayoría en la lengua materna con muy poco uso del idioma inglés en este caso. El docente no tiene que hablar inglés bien, solo se utilizan libros textos y los estudiantes sienten menos stress pues las clases se desarrollan en la lengua materna. A pesar de que presenta estas ventajas, las desventajas los superan dado que los estudiantes solo comprenden palabra por palabra, no aprenden a leer, solo utilizan la memorización y hay muy poca interacción entre estudiante y docente. Este método difiere de la traducción pedagógica.

\section{La comprensión lectora}

Harrison (2004) manifiesta que la comprensión es el acto o el hecho de comprender con la mente; es decir, entender. Es la habilidad de entender un texto y contestar preguntas sobre él. Mientras que para Pouya (2012) la lectura es un proceso por el cual se amplía el conocimiento sobre alguna materia en el idioma que se desea aprender. Se lee con el objetivo de adquirir la habilidad de leer revistas y libros en ese idioma que en este caso es inglés; es la clave para que el lector investigue la mente de otros, comparta lo que han aprendido y sientan lo que han sentido.

Leer de manera efectiva en un idioma extranjero es una de las actividades más desafiantes que muchos estudiantes deben enfrentar en las escuelas. Esta destreza ayuda a que los estudiantes tengan éxito en el proceso de aprendizaje tanto en su vida personal y escolar (Dechant, 1991).

\section{La comprensión lectora de textos académicos}

El inglés para propósitos específicos se divide en tres variantes: inglés para propósitos académicos (con fines académicos), Ingles para propósitos ocupacionales (para fines profesionales) e inglés para ciencia y tecnología (para ciencia y tecnología). (Hernández Medina, 2003)

El inglés para propósitos específicos cubre una amplia variedad de contenidos como administración, medicina, leyes, ingeniería, historia y arte y diseño. De hecho, cualquier área de la vida profesional y académica moderna en la cual se necesita el idioma inglés. El inglés para propósitos específicos no es un enfoque, método o técnica. La principal característica que tienen en común todos los cursos de inglés para propósitos específicos es la selección del contenido y el enfoque de acuerdo a las necesidades de los estudiantes. En tal virtud, el 
análisis de las necesidades juega un papel mucho más importante que en el inglés general. Inglés para propósitos específicos se refiere a la enseñanza del inglés para los estudiantes que están aprendiendo el idioma para realizar un trabajo en particular o por razones relacionadas a sus estudios. (Tomlinson, 2013). Ahora bien, el inglés para fines académicos se imparte dentro del mismo lugar de estudio y tiene como objetivo satisfacer los requerimientos lingüísticos de los estudiantes; es así como ellos pueden desenvolverse dentro y fuera de clases con respecto a sus estudios. (Hernández Medina, 2003).

Cuando los estudiantes leen, lo hacen con un propósito el cual puede incluir varios propósitos: obtener información, entender ideas o teorías, descubrir los puntos de vista de los autores, buscar evidencia que contribuyan sus propios puntos de vista. Dentro de este proceso, los estudiantes tendrán que preocuparse tanto del contenido de la materia como del idioma en el cual está escrito el texto. Ambos aspectos requieren comprensión, pero de diferente tipo. (Jordan, 1997).

En cuanto a los propósitos de la lectura académica (Lolino, 2014) indica que el propósito es un elemento crucial pues no ayuda a determinar el tipo de lectura y las estrategias que se utilizarán, es así que el objetivo de la lectura se ha alcanzado de manera exitosa. Este autor citando a Grabe (2009:8) señala que los muchos propósitos de las lecturas son: leer por placer, entrenamiento, leer para aprender y leer para obtener información.

\section{Textos especializados}

En cuanto al adjetivo especializado, sencillamente es especializado porque no es general en la parte comunicativa y no es de dominio común. El texto es una unidad comunicativa de carácter lingüística a través del cual se transmite significados. La definición de texto especializado surge de la distinción entre lenguaje especializado y lenguaje general. El lenguaje general comprende reglas lingüísticas utilizadas por los individuos para comunicarse. El lenguaje especializado es el uso de todos los recursos lingüísticos en el ámbito comunicativo, es decir, la temática, el tipo de interlocutores, la situación comunicativa, la intencionalidad del emisor, etc., a fin de que las personas que laboran en ese ámbito comprendan el texto. (Guantiva Acosta, Cabré M. T., \& Castellá, 2008)

Un texto especializado tiene unidades lingüísticas y comunicativas y una temática especializada. 


\section{Características de los textos especializados}

Guantiva Acosta (2008) pone de manifiesto que el texto se caracteriza por ser una unidad estructural compuesto de oraciones coherentes pragmáticas, semánticas y sintácticas. Debido a su compleja estructura comunicativa, el texto se ve afectado por los siguientes factores:

- Del autor y estrategia comunicativa derivada de ella

- Destinatario

- Léxico de acuerdo a la terminología que puede presentar grados de especialización y variación expresiva. Cabré 1999: 156-159

El texto está sujeto a factores sociopragmáticos que afectan su tipología y estos son:

- La temática

- El tipo de interlocutor

- Las circunstancias comunicativas según el registro empleado.

Un texto es especializado si reúne criterios lingüísticos, cognitivos y pragmáticos. Desde el punto de vista textual, se caracterizan por una configuración global específica y un estilo con variaciones según la temática y los niveles en los que se tratan.

Desde el punto de vista léxico, los textos especializados cuentan con una gran cantidad de términos y estos aumentan o disminuyen según el grado de especialidad.

Desde el punto de vista funcional, su función primordial es la transmisión de información y la terminologia denomina conceptos de una lengua especializada. El texto especializado como unidad se basa en:

- Propiedades textuales generales (componente semántico y formal general)

- Dependencia al ambito tematico (lexico y combinaciones léxicas o fraseologia recurrente)

- Uso de unidades de otros sistemas que se integran en el discurso de especialidad.A nivel estructural se caracterizan por el nivel textual (precisos, concisos y sistematicos) y gramatical (terminologia del tema) 
Desde el punto de vista cognitivo, los textos especializados tienen que ver con un conocimiento preciso resultante de un proceso de conceptualizacion de la realidad validado por especialistas del ambito.

Desde el punto de vista tematico, el texto es especializado si correponde a una de las siguientes disciplinas:

- Materias científico técnicas

- Ciencias exactas

- Ciencias experimentales

- Ciencia sociales

- Ciencias humanas

- Profesiones

- Actividades especializadas

Desde el punto de vista funcional, su funcion primordial es la transmisión de información y la función de la terminología es denominar conceptos en una area de especialización.

Cabré y Enterría (Cab06) mencionan sobre las lenguas de especialidad que:

“.....como subconjuntos de recursos especificos, lingüisticos y no lingüísticos, discursivos y gramaticales, que se utilizan en situaciones consideradas especializadas por sus condiciones comunicativas".

Las características del texto especializado desde el punto de vista de la producción, se analizan desde tres elementos:

- Las características previas a la producción del texto

- La programación del texto

- Elaboración del texto.

\section{Variación discursiva}

Es la adecuación del texto según el contexto. El contexto es el campo de cultivo del cual se desprende el texto. En este proceso intervienen factores funcionales, situacionales y temáticos que generan registros, tipos y géneros textuales. 
Los registros son formas lingüísticas determinadas, condicionadas por funciones comunicativas concretas. Los registros orientan al interlocutor al momento de producir el texto y al receptor en sus expectativas del texto que va a interpretar. Los elementos de la realidad contextual que interviene en los registros se agrupan de tres formas:

- El campo o tema permite hacer la distinción entre temática general (campos de estudio) y temática especializada (grados o niveles de abstracción o especialización con un solo campo)

- El tenor permite la relación entre emisor y receptor tomando en cuenta el grado de formalidad, intencionalidad del autor, objetivo.

- El modo es el medio por el cual se emite, transmite y recibe el mensaje.

Alcina Caudet (2005) afirma que el género es una herramienta para el traductor ya que este puede hacer uso de la clasificación en género de los textos para poder solucionar conflictos de traducción.

Tipo textual, registro y género

El tipo textual determina el registro y el género de un texto. También determina el propósito retórico que puede ser expositivo, argumentativo y exhortativo. El registro explica que el lenguaje se utiliza de manera diferente dependiendo de la situación. Reúne las características lingüísticas del texto resultantes de tres factores situacionales del texto que convergen en la producción del texto: tenor, campo y modo. Además, presenta restricciones léxicas y sintácticas. El género presenta restricciones en la estructura discursiva del texto. El género explica como el hablante de una lengua puede identificar características y funciones presentes en determinados textos relacionados con situaciones sociales habituales en una cultura. (López Rodríguez, 2000)

\section{Clasificación de los textos especializados}

Los textos especializados se clasifican según el nivel de especialización: textos muy especializados, semi-especializados y de divulgación. Y los especializados ningún especialista intervienes.

La especialización de la lengua está relacionada con la terminología, por eso a las palabras se las denomina como términos. El lenguaje científico hace uso de la lengua común, pero 
dependiendo del ámbito se crean nuevos términos para determinar nuevas realidades. En la medicina y la jurisprudencia los términos se encuentran más normalizados. (Anguita, 2002)

\section{Conclusiones}

La traducción es un recurso didáctico que de ser utilizado de manera adecuada por los docentes y escogido el material en base a las necesidades de los estudiantes es beneficioso para ayudar a desarrollar la destreza lectora de los estudiantes universitarios quienes por sus actividades formativas se enfrentan día a día a tipos de texto de carácter especializado cuyas características requieren ser entendidas e identificadas para poder comprender su contenido. Los estudiantes no solo tienen el reto de entender el contenido de carácter académico, sino que a la vez deben hacerlo en un idioma que no es el materno. Este tipo de actividades de traducción no se basan en el método de grammar-translation que es muy a menudo menospreciado por los docentes de inglés. El método de grammar-translation se centra en el docente mientras que las actividades de traducción que se deben desarrollar con los estudiantes deben estar centradas en ellos.

Las actividades de traducción centradas en los estudiantes pueden ayudar a mejorar la comprensión lectora de los estudiantes universitarios. Es necesario que estos sean conscientes del tipo de texto y sus características para poder entender lo que leen de manera efectiva.

Las características de los textos especializados requieren de un tratamiento diferente al que se le daría a un texto de lenguaje general.

\section{Referencias bibliográficas}

Alcina Caudet, A. (2005). La implementación del concepto de género textual en los corpus electrónicos para traductores. Obtenido de http://tecnolettra.uji.es/pubs/Alcina_05_El_genero_textual_y_la_traduccion.pdf

Anguita, J. M. (2002). La traducción científico-técnica: situación actual en España. Obtenido de http://cvc.cervantes.es/lengua/esletra/pdf/01/026_anguita.pdf

Byrne, J. (2012). Scientific and Technical Translation Explained. A Nuts and Bolts Guide for Beginners. New York: Routledge.

Catford, J. (1995). A linguistic Theory of Translation. . London: Oxfored University Press. 
Dechant, E. (1991). Understanding and teaching reading: An interacttive model. Hillsdale, NJ: Lawrence Erlbaum. Hillsdale: L. Erlbaum Associates.

Ghazala, H. (1995). Translation as Problems and Solutions. Syria.

Guantiva Acosta, R., Cabré M. T., \& Castellá, J. (2008). Clasificación de los textos especializados a partir de su terminología. Obtenido de http://www.redalyc.org/articulo.oa?id=255020449002

Harrison, C. (2004). Understanding Reading Development. Great Britain: SAGE Publications.

Hernández Medina, J. J. (2003). Los análisis del género, discurso y registro en el alemán para fines turísticos. Almería: Universidad de Almeria.

Hernández, M. R. (1996). La traducción pedagógica en la clase de E/LE. Centro Virtual Cervantes, 2.

Jordan, R. R. (1997). English for Academic Purposes: A Guide and Resource Book for Teachers. Cambridge: Cambridge University Press.

Leonardi, V. (2010). The role of pedagogical translation in second language acquisition. From theory to practice. Great Britain: The British Library.

Lolino, A. (2014). Reading for Academic Purposes: Techniques and Strategies to help Angolan ELT Students at ISCED-Benguela enhance their reading skills. Universidad Nova de Lisboa. Obtenido de https://pdfs.semanticscholar.org/abc7/03aa9e6464f40a844d63e9222086cb4cfdd8 .pdf

Lonsdale, A. (1993). Teaching Translation from Spanish to English: Worlds Beyond Words. Canada: University of Ottawa Press.

López Rodríguez, C. I. (2000). Tipologías textuales y géneros en la normalización terminológica y ortotipográfica de la traducción médica. Obtenido de http://www.ugr.es/ clarailr/lopez_2000_tipologias_generos.pdf

Loranc-Paszylk, B. (2019). Rethinking directions in language learning and teaching at university level. France: Research-publishing.net.

Mahmoud, A. (2006). Translation and Foreign Language Reading Comprehension: A Neglected Didactic Procedure. Engglish teaching forum. 
Pouya, F. (2012). On The Effect of Translation on Promoting Reading Comprehension in ESP. Journal of Basic and Applied Scientific Research, 8.

Shei, C., \& Gao, Z.-M. G. (2017). The Routledge Handbook of Chinese Translation. London.

Tomlinson, B. (2013). Developing Materials for Language Teaching. London: Bloomsbury Academic. 
Para citar el artículo indexado.

Barragán Murillo, R. de los Ángeles. (2019). El uso de la traducción para mejorar la comprensión lectora de textos especializados en los estudiantes universitarios. Explorador Digital, 3(3), 156168. https://doi.org/10.33262/exploradordigital.v3i3.883

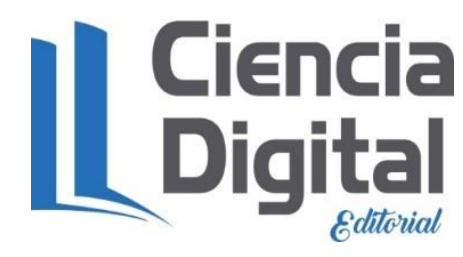

El artículo que se publica es de exclusiva responsabilidad de los autores y no necesariamente reflejan el pensamiento de la Revista Explorador Digital.

El articulo queda en propiedad de la revista y, por tanto, su publicación parcial y/o total en otro medio tiene que ser autorizado por el director o editor de la Revista Explorador

Digital.
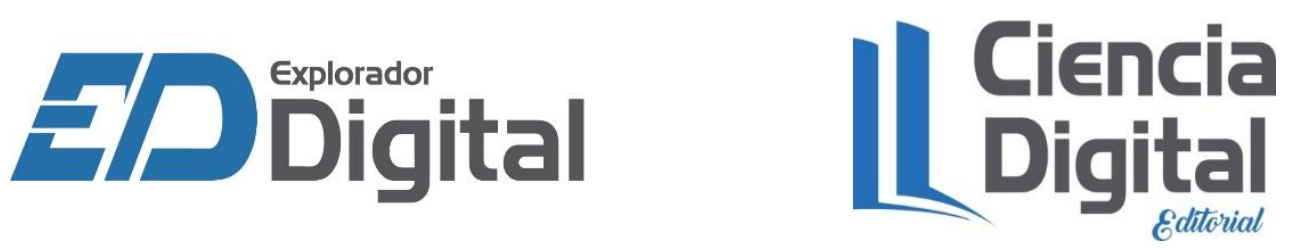